\title{
ON LOCAL AND GLOBAL REGULARITY OF FOURIER INTEGRAL OPERATORS
}

\author{
MICHAEL RUZHANSKY
}

\begin{abstract}
The aim of this paper is to give a review of local and global properties of Fourier integral operators with real and complex phases, in local $L^{p}$, global $L^{2}$, and in Colombeau's spaces.
\end{abstract}

\section{INTRODUCTION}

Fourier integral operators and their regularity properties have been under study since their appearance in [22]. One of the main motivations comes from the Cauchy problem for hyperbolic partial differential equations. Consider a pseudo-differential operator

$$
P\left(t, x, \partial_{t}, \partial_{x}\right)=\partial_{t}^{m}+\sum_{j=1}^{m} P_{j}\left(t, x, \partial_{x}\right) \partial_{t}^{m-j}
$$

of order $m$. Here $t \in \mathbb{R}$ and $x \in X$, where $X \subset \mathbb{R}^{n}$ is an open set. Operators $P_{j}(t, \cdot, \cdot) \in \Psi^{j}(X)$ are assumed to be (classical) pseudo-differential operators of order $j$ on $X$.

Let $\sigma_{P}(t, \tau, x, \xi)$ be the principal symbol of $P$, i.e. the top order part of the full symbol of $P$, positively homogeneous of degree $m$ in $\xi$. As usual, $\tau$ and $\xi$ correspond to $D_{t}=-i \partial_{t}$ and $D_{x}=-i \partial_{x}$, respectively.

Let $P$ be strictly hyperbolic, i.e. assume that $\sigma_{P}$ can be factored as

$$
\sigma_{P}(t, x, \tau, \xi)=\prod_{j=1}^{m}\left(\tau-\tau_{j}(t, x, \xi)\right),
$$

where $\tau_{j}(t, x, \xi)$ are real and distinct for $\xi \neq 0$. Then they are positively homogeneous of degree one in $\xi$ and smooth in $(t, x)$, for $\xi \neq 0$. It is well-known that under the strict hyperbolicity condition the Cauchy problem for $P$ is well-posed. The Cauchy problem for $P$ is the equation

$$
\begin{cases}P u(t, x)=0, & t \neq 0, \\ \left.\partial_{t}^{j} u\right|_{t=0}=f_{j}(x), & 0 \leq j \leq m-1 .\end{cases}
$$

The loss of regularity for solutions $u(t, \cdot)$ compared to the Cauchy data depends on the operator $P$ and on the function spaces. For example, the usual energy conservation that holds in $L^{2}$ fails in $L^{p}$ for $p \neq 2$.

Date: December 28, 2007.

1991 Mathematics Subject Classification. Primary 35S30; Secondary 35L30.

Key words and phrases. Fourier integral operators, hyperbolic partial differential equations.

The author was supported by a Royal Society grant and by the EPSRC Grant EP/E062873/01. 
The general question that one is interested in is as follows. Let $W_{j}, W$ be some function spaces. The questions is whether for (small) $t$ we have the property that

$$
f_{j} \in W_{j}(\text { for all } j) \Longrightarrow u(t, \cdot) \in W \quad ?
$$

First, recall the following examples of such mapping properties:

$$
f_{j} \in L_{n-j}^{2} \Longrightarrow u(t, \cdot) \in L_{n}^{2}
$$

and

$$
f_{j} \in L_{n-j+(n-1)|1 / p-1 / 2|}^{p} \Longrightarrow u(t, \cdot) \in L_{n}^{p} .
$$

Here and in the sequel $L_{s}^{p}$ will denote the local Sobolev space over $L^{p}$ defined by the property that $f \in L_{s}^{p}$ if $(1-\Delta)^{s / 2} f \in L^{p}$.

Property (1.2) is the usual conservation of energy for hyperbolic equations in $L^{2}$. Property (1.3) shows that the loss of regularity occurs in $L^{p}$ for $p \neq 2$. In the case of the wave equation this was established in [56], [33], [36]. In general, this property was proved in [54] (see also [55] and [57]). We note that this is quite different from $L^{p}-L^{p^{\prime}}$ properties, for some overview of which see e.g. [47].

The main idea for deriving estimates (1.3) is to use the fact that solution $u(t, x)$ to the Cauchy problem (1.1) can be written as a sum of Fourier integral operators (see e.g. [30], [15], 24], [16]). Thus, the study of the regularity properties of solutions to the Cauchy problem (1.1) are reduced to the analysis of the regularity properties of Fourier integral operators. A more complicated reduction is also possible for certain classes of hyperbolic systems, even with variable multiplicities, for example for systems with microlocally diagonalisable principal part (see [38], and [26] for generic conditions on characteristics).

\section{Fourier INTEGRAL OPERATORS WITH REAL PHASES}

Let $X, Y$ be open sets in $\mathbb{R}^{n}$. One defines the class of Fourier integral operators $T \in I^{\mu}\left(X \times Y, C^{\prime}\right)$ by the (microlocal) formula

$$
T f(x)=\int_{Y} \int_{\mathbb{R}^{n}} e^{i \Phi(x, y, \theta)} a(x, y, \theta) f(y) d \theta d y,
$$

where amplitude $a$ is a smooth function satisfying

$$
a \in S^{\mu}:\left|\partial_{x, y}^{\alpha} \partial_{\theta}^{\beta} a(x, y, \theta)\right| \leq C_{\alpha \beta}(1+|\theta|)^{\mu-|\beta|},
$$

for all multi-indices $\alpha, \beta$, and $\Phi$ is a phase function. First, we assume that the phase is real-valued and satisfies the following properties:

1. $\Phi(x, y, \lambda \theta)=\lambda \Phi(x, y, \theta)$, for all $\lambda>0$;

2. $d \Phi \neq 0$;

3. $d_{\theta} \Phi=0$ is smooth (e.g. $d_{\theta} \Phi=0$ implies $d_{(x, y, \theta)} \frac{\partial \Phi}{\partial \theta_{j}}$ are independent).

It turns out that Fourier integral operators have a useful invariant quantity, the canonical relation, which is a conic Lagrangian manifold in $\left(T^{*} X \backslash 0\right) \times\left(T^{*} Y \backslash 0\right)$, equipped with the symplectic form $\sigma_{X} \oplus-\sigma_{Y}$, where $\sigma_{X}$ and $\sigma_{Y}$ are canonical symplectic forms in $T^{*} X$ and $T^{*} Y$, respectively. The canonical relation of a Fourier integral operator $T$ is defined by $C^{\prime}=\{(x, \xi, y,-\eta):(x, \xi, y, \eta) \in C\}$, where

$$
C=C_{\Phi}=\left\{\left(x, d_{x} \Phi, y, d_{y} \Phi\right): d_{\theta} \Phi=0\right\}
$$


is the wave front set of the integral kernel of the operator $T$. We have the canonical projections:

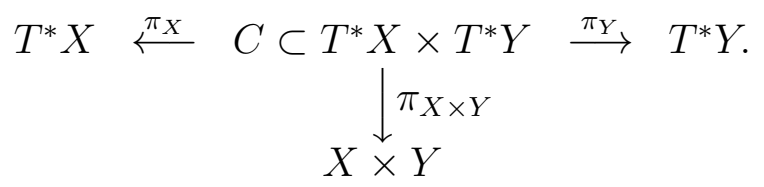

Operators appearing in the analysis of hyperbolic equations satisfy the so-called local graph condition. It means that projections

$$
\pi_{X}: C \rightarrow T^{*} X \backslash 0, \quad \pi_{Y}: C \rightarrow T^{*} Y \backslash 0
$$

are local symplectic diffeomorphisms. In fact, because of the symplectic structure, one of them is diffeomorphic if and only if the other one is. In terms of the phase function $\Phi$, it means that we have

$$
\operatorname{det} \partial_{y} \partial_{\theta} \Phi \neq 0
$$

or similarly $\operatorname{det} \partial_{x} \partial_{\theta} \Phi \neq 0$. Thus, from now on we will assume that Fourier integral operators that we consider are non-degenerate, i.e. that the local graph condition is satisfied. Note that the degenerate case is quite more subtle, see e.g. [37] for some overview.

The local $L^{2}$-boundedness of non-degenerate Fourier integral operators of order zero is well-known ([17], [22]). In $L^{p}$-spaces with $p \neq 2$ there is a loss of regularity depending on the index $p$. Indeed, it was shown in [54] that non-degenerate Fourier integral operators of order $\mu$ are locally bounded in $L^{p}, 1<p<\infty$, provided that $\mu \leq$ $-(n-1)|1 / p-1 / 2|$. This follows by interpolation between the local $L^{2}$-boundedness of Fourier integral operators of order zero and local boundedness of Fourier integral operators of order $-(n-1) / 2$ from the Hardy space $H^{1}$ to $L^{1}$, established in [54]. Alternatively, it was also established in [60] that non-degenerate Fourier integral operators of order $-(n-1) / 2$ are of weak $(1,1)$-type. In Section 4 we will discuss the local $L^{p}$-properties further.

\section{Fourier INTEGRAL OPERATORS With COMPLEX PHASES}

Let us now briefly describe Fourier integral operators with complex phase functions. Again, let $X, Y$ be open subsets of $\mathbb{R}^{n}$. Then a Fourier integral operator of order $\mu$ with complex phase is defined by the same formulae as in (2.1), namely

$$
T f(x)=\int_{\mathbb{R}^{n}} \int_{\mathbb{R}^{n}} e^{\mathrm{i} \Phi(x, y, \theta)} a(x, y, \theta) f(y) d \theta d y,
$$

where amplitude $a \in S^{\mu}$ is of order $\mu$ and the complex-valued phase function $\Phi \in \mathbb{C}$ satisfies conditions:

1. $\Phi(x, y, \lambda \theta)=\lambda \Phi(x, y, \theta)$, for all $\lambda>0$

2. $d \Phi \neq 0$;

3. $d_{\theta} \Phi=0$ is smooth (e.g. $d_{\theta} \Phi=0$ implies $d \frac{\partial \Phi}{\partial \theta_{j}}$ are independent over $\mathbb{C}$ );

4. $\operatorname{Im} \Phi \geq 0$.

The last property is clearly necessary in general, for the integral (3.1) to be welldefined. The theory of Fourier integral operators with complex phases was developed 
by Melin, Sjöstrand in [31] and [32], see also [24] and [61] for slightly alternative descriptions. These operators are also related to the Maslov canonical operator.

In [29], Laptev, Safarov and Vassiliev established global parameterisations of Fourier integral operators with real phases by globally defined complex phases (see also [53]).

Again, the canonical relation of $T$ with complex phase $\Phi$ is defined by

$$
C=C_{\Phi}=\left\{\left(x, d_{x} \Phi, y, d_{y} \Phi\right): d_{\theta} \Phi=0\right\} .
$$

Now we can identify $C$ with a subset of $T^{*} \widetilde{(X \times Y)}$, where $T^{*} \widetilde{(X \times Y)}$ is an almost analytic extension of $T^{*}(X \times Y)$. Here one needs to work with almost analytic extensions of real sets, for example in order to interpret the eikonal equation $\partial_{t} \Phi=$ $\tau\left(t, x, \nabla_{x} \Phi\right)$ when $\Phi$ is complex-valued.

Local $L^{2}$ properties for operators with complex phases are more subtle. For example, in [32], Melin and Sjöstrand showed that operators of order zero are locally bounded in $L^{2}$ under a complex-valued version of a local graph condition. This condition and its extension will be discusses in the next section in more detail. A more general result was established by Hörmander in [23]. Assume that for every $\gamma \in C_{\mathbb{R}}$, the maps $\left(T_{\gamma} C\right)_{\mathbb{R}}$ to $T_{\pi_{X}(\gamma)} T^{*} X$ and to $T_{\pi_{Y}(\gamma)} T^{*} Y$ are injective. Then it is shown in 23] that Fourier integral operators of order zero with complex-valued phases are locally bounded in $L^{2}$. In fact, one can shows that if the mappings

$$
C_{\mathbb{R}} \rightarrow T^{*} X \backslash 0, \quad C_{\mathbb{R}} \rightarrow T^{*} Y \backslash 0
$$

are injective, then $T \circ T^{*} \in \Psi_{1 / 2,1 / 2}^{0}$ is a pseudo-differential operator of order zero and type $(1 / 2,1 / 2)$. This is one of the differences with operators with real-valued phases, where $T \circ T^{*} \in \Psi_{1,0}^{0}$ is a pseudo-differential operator of order zero and type $(1,0)$.

\section{LOCAL $L^{p}$ REGULARITY PROPERTIES OF FOURIER INTEGRAL OPERATORS}

The local $L^{p}$ properties of Fourier integral operators can be summarised in the following table:

\begin{tabular}{|l||c|c|}
\hline & $\Phi \in \mathbb{R}$ & $\Phi \in \mathbb{C}$ \\
\hline$L^{2}$ & {$[17,22]$} & {$[31,32,23]$} \\
\hline$L^{p}$ & {$[54]$} & {$[42]$} \\
\hline
\end{tabular}

To explain this is more detail, we have the following results for the local $L^{p}$ continuity:

- Real $L^{2}$ theory: Eskin [17] and Hörmander [22]: operators of order zero with real phases are locally continuous in $L^{2}$.

- Real $L^{p}$ theory: Stein [56], Beals [4], Miyachi [33], Peral [36], Sugimoto [59], and finally Seeger, Sogge and Stein [54]: operators of order $\mu$ with real phases are locally continuous in $L^{p}$, provided that $\mu \leq-(n-1)|1 / p-1 / 2|$.

- Complex $L^{2}$ theory: Melin and Sjöstrand [31, 32] and Hörmander [23]: operators of order zero with complex phases are locally continuous in $L^{2}$.

- Complex $L^{p}$ theory: Ruzhansky [42]: extension of the properties above to derive the unified local $L^{p}$ properties for operators with complex phases.

We will now review local $L^{p}$ results for Fourier integral operators with complex phases in more detail. 
Let us introduce the following local graph condition for complex-valued phases. We will assume that there exists some $\tau \in \mathbb{C}$ such that we have

$$
\operatorname{det} \partial_{\theta} \partial_{y}(\operatorname{Re} \Phi+\tau \operatorname{Im} \Phi) \neq 0 \text {. }
$$

Since the equation of the determinant to zero is polynomial in $\tau$, we can conclude that this condition is equivalent to the existence of $\lambda \in \mathbb{R}$ such that

$$
\Psi=\operatorname{Re} \Phi+\lambda \operatorname{Im} \Phi
$$

defines a (real) local graph, i.e. that $\operatorname{det} \partial_{\theta} \partial_{y} \Psi(x, y, \theta) \neq 0$. Condition (LG) weakens Melin-Sjöstrand's local graph condition, which corresponds to the case $\tau=i$. Following [42, under this condition we have the following results. For the sake of simplicity in notation we will suppress primes in the notation for canonical relations.

Theorem 4.1 ([42]). Let $C \subset\left(T^{*} X \backslash 0\right) \times\left(T^{*} Y \backslash \widetilde{0}\right)$ be a smooth complex positive homogeneous canonical relation, closed in $T^{*}(\widehat{X \times Y}) \backslash 0$. Assume that $(L G)$ holds. Let $\mu=-(n-1)|1 / p-1 / 2|, 1<p<\infty$. Then $T \in I^{\mu}\left(X \times Y, C^{\prime}\right)$ is continuous from $L_{\text {comp }}^{p}(Y)$ to $L_{l o c}^{p}(X)$.

This theorem extends both the result of Melin and Sjöstrand in [31, 32] $\left(L^{2}, \Phi \in \mathbb{C}\right)$ and the result of Seeger, Sogge and Stein in [54] $\left(L^{p}, \Phi \in \mathbb{R}\right)$. We can also note that Theorem 4.1 can not be obtained from its real-valued version since we only have that there exists a real conic Lagrangian manifold $C_{0} \subset T^{*}(X \times Y) \backslash 0$ such that $C_{\mathbb{R}} \subset C_{0}$ and such that the class of operators with complex phase of order $\mu$ with canonical relation $C$ is contained in the class of operators of the same order $\mu$ with real phases with real canonical relation $C_{0}$ of type $(1 / 2,1 / 2)$, i.e. we have $I_{\rho}^{\mu}(X, Y ; C) \subset$ $I_{1 / 2}^{\mu}\left(X, Y ; C_{0}\right)$. Thus, we may only conclude that operators with complex phases of order $\mu$ are locally bounded in $L^{p}$ provided that $\mu \leq-(n-1 / 2)|1 / p-1 / 2|$. This order is clearly worse than the order $\mu$ given in in Theorem 4.1,

\section{Cauchy Problem For operators With COMPleX CHARACTERISTiCS}

The results can be applied to establish the local regularity properties to solutions to the Cauchy problem for operators with complex characteristics. Consider a (classical) pseudo-differential operator

$$
P\left(t, x, D_{t}, D_{x}\right)=D_{t}^{m}+\sum_{j=1}^{m} P_{j}\left(t, x, D_{x}\right) D_{t}^{m-j}
$$

of order $m$, where $t \in[0, T]$ and $x \in X \subset \mathbb{R}^{n}$. Here $P_{j} \in C^{\infty}\left([0, T], \Psi_{c l}^{j}(X)\right)$ are classical pseudo-differential operators of order $j$.

The principal symbol of operator $P$ is given by

$$
\sigma_{P}(t, x, \tau, \xi)=\tau^{m}+\sum_{j=1}^{m} P_{j}(t, x, \xi) \tau^{m-j}=\prod_{j=1}^{m}\left(\tau-\tau_{j}(t, x, \xi)\right) .
$$

The Cauchy problem for operator $P$ is the equation

$$
\begin{cases}P u(t, x)=0, & t \neq 0 \\ \left.\partial_{t}^{j} u\right|_{t=0}=f_{j}(x), & 0 \leq j \leq m-1\end{cases}
$$


It was shown in [61] that the propagator for this Cauchy problem can be expressed in terms of Fourier integral operators with complex phases, under the assumption that characteristic roots $\tau_{j} \in \mathbb{C}$ are complex-valued and satisfy the following assumptions (A1) and (A2):

\section{(A1) Simple characteristics: $\tau_{i} \neq \tau_{j}, i \neq j, \xi \neq 0$.}

Therefore, we have that $\tau_{j} \in C^{\infty}\left([0, T] \times\left(T^{*} X \backslash 0\right), \mathbb{C}\right)$ is smooth and positively homogeneous of order one in $\xi$. We also assume that

$$
(A 2): \quad \operatorname{Im} \tau_{j}(t, x, \xi) \geq 0 \text { in }[0, T] \times\left(T^{*} X \backslash 0\right) .
$$

Theorem 5.1 ([42]). Let $P$ satisfy (A1) and (A2). Let $1<p<\infty$. Let $f_{j}$ be compactly supported. Then we have locally:

$$
f_{j} \in L_{\alpha-j+(n-1)|1 / p-1 / 2|}^{p}(j=0, \ldots, m-1) \Longrightarrow u(t, \cdot) \in L_{\alpha}^{p} .
$$

Also, there exists a constant $C_{T}$ such that for all $t \in[0, T]$ we have the local estimate

$$
\|u(t, \cdot)\|_{L_{\alpha}^{p}} \leq C_{T} \sum_{j=0}^{m-1}\left\|f_{j}\right\|_{L_{\alpha-j+(n-1)|1 / p-1 / 2|}^{p}} .
$$

The result is sharp in general, since the loss of $(n-1)|1 / p-1 / 2|$ derivatives is sharp for wave type equations (where $\tau_{j} \in \mathbb{R}$ ).

We note that the regularity results for operators with complex-valued functions have other applications, see e.g. [42] for an application to the oblique derivative problem (see also [45] and [42]).

\section{Sharpness of LOCAL $L^{p}$ PROPERTIES}

Let $T \in I^{\mu}(X, Y ; C)$ be a Fourier integral operator with a real-valed phase function $\Phi(x, y, \theta)$. The question for the sharpness of local $L^{p}$ estimates is to find the largest $\mu$ for which operators of order $\mu$ are continuous from $L_{\text {comp }}^{p}$ to $L_{l o c}^{p}$. Alternatively, by using the calculus, one can look for the smallest $\alpha$ such that operators of order zero are continuous from the Sobolev space $L_{\alpha}^{p}$ to $L^{p}$. In this case we have $\mu=-\alpha$.

Let

$$
k:=\max _{x, y, \theta} \operatorname{rank} \frac{\partial^{2}}{\partial \theta^{2}} \Phi(x, y, \theta) .
$$

In particular, the meaning of $k$ is that the dimension of the singular support of the integral kernel of $T$ is less or equal to $n+k$ (see [40]). For example, we have $k=n-1$ for the solutions of the wave equation and $k=0$ for pseudo-differential operators. The following result was proved in [39]:

Theorem 6.1 ([39]). If a Fourier integral operator $T$ of order zero with real phase is locally bounded from $L_{\alpha}^{p}$ to $L^{p}$, then $\alpha \geq \alpha_{p}=k|1 / p-1 / 2|$.

There are the following important cases of this result:

- $p=2$; here $\mu=0$.

- $1<p<\infty$ and $k=n-1$ : this is the case of solutions to the wave equations ([36]) and more general hyperbolic equations ([54]); here $\mu=-(n-1) \mid 1 / p-$ $1 / 2 \mid$. 
- $k=0$ : this is the case of pseudo-differential operators; here $\mu=0$.

The orders in Theorem 6.1 indicate that positive results on the $L^{p}$ boundedness and the order $\mu$ in Theorem 4.1 can be improved if we impose further conditions on the canonical relation $C$.

Let us first review such positive results for operators with real-valued phases. Define

$$
\Sigma=\left\{(x, y): \operatorname{rank} \frac{\partial^{2}}{\partial \theta^{2}} \Phi(x, y, \theta)=k\right\}
$$

If $(x, y) \in \Sigma$, the level set of $\nabla_{\theta} \Phi$ is a linear space of dimension $n-k$ in $C_{\Phi} \subset$ $T^{*}(X \times Y)$, corresponding to a linear subspace in the conormal bundle

$$
\begin{aligned}
N^{*} \Sigma=\{ & (x, y, \xi, \eta) \in T^{*}(X \times Y):(x, y) \in \Sigma, \\
& \left.\xi(\delta x)+\eta(\delta y)=0, \forall(\delta x, \delta y) \in T_{(x, y)} \Sigma\right\} .
\end{aligned}
$$

The inclusion $N^{*} \Sigma \subset C_{\Phi}$ is dense, and the conormal bundle $N^{*} \Sigma$ consists of affine fibers.

In [54], the following so-called smooth factorization condition (SF) was introduced. Assume that the mapping

$$
(x, y) \mapsto\left(\theta-\text { level set of } \nabla_{\theta} \Phi(x, y, \theta)\right): \Sigma \rightarrow \mathbb{G}_{n-k}\left(\mathbb{R}^{n}\right)
$$

is smoothly extendible from $\Sigma$ to $\pi_{X \times Y}\left(C_{\Phi}\right)$, the singular support of the integral kernel of operator $T$ with canonical relation $C_{\Phi}$. Here $\mathbb{G}_{n-k}\left(\mathbb{R}^{n}\right)$ denoted the Grassmanian which is the collection of all $(n-k)$-dimensional linear subspaces of $\mathbb{R}^{n}$.

We can note the the smooth factorization condition (SF) automatically holds in the case $k=0$ (pseudo-differential operators) and in the case $k=n-1$ (hyperbolic equations).

Theorem $6.2([54])$. Under the smooth factorization condition (SF) operators Fourier integral operators of order zero with real phases are locally continuous from $L_{\alpha_{p}}^{p}$ to $L^{p}$, provided that $\alpha_{p}=k|1 / p-1 / 2|$ and $1<p<\infty$.

Thus, a question arises when and whether the smooth factorization condition (SF) is satisfied. One answer is given by the following theorem:

Theorem 6.3 ([40]). For translation invariant Fourier integral operators with analytic phases, the smooth factorization condition $(\mathrm{SF})$ is satisfied, provided that $n \leq 4$, or $k \leq 2$.

As a consequence, one obtains a sharp list of $L^{p}$-properties for translation invariant Fourier integral operators in $\mathbb{R}^{n}$, with $n \leq 4$, or $k \leq 2$. The analysis is based on the theory of affine fibrations developed in [41].

Theorem 6.3 shows that in order for the smooth factorization condition to break, one needs to have the dimension at least $n \geq 5$. In such case examples for the failure of (SF) have been constructed in [39] and in [41]. A typical example is the phase function defined by

$$
\Phi(x, y, \theta)=\langle x-y, \theta\rangle+\theta_{1} \theta_{2}^{2} \theta_{5}^{-2}+\left(\theta_{3} \theta_{5}-\theta_{2} \theta_{4}\right)^{2} \theta_{5}^{-3} .
$$

If the operator is not translation invariant, one can construct simpler examples already in lower dimensions. For example, if $n=3$, the phase function

$$
\Phi(x, y, \theta)=\langle x-y, \theta\rangle+\theta_{3}^{-1}\left(y_{1} \theta_{1}+y_{2} \theta_{2}\right)^{2}
$$


provides an example of the failure of $(\mathrm{SF})$. However, in [44] it was shown that the loss of regularity for the corresponding Fourier integral operators is the same as for operators satisfying the smooth factorization condition (SF).

An analogue of the smooth factorization condition for complex phases was discussed in [42. The complex-valued phase function $\Psi$ satisfies the complex smooth factorization condition ( $\mathbb{C S F}$ ) if there exists a real $|\tau|<1 / \sqrt{3}$ such that:

- $\Psi=\operatorname{Re} \Phi+\tau \operatorname{Im} \Phi$ satisfies (LG);

- $\Psi$ satisfies real $(\mathrm{SF})$ for some $k$.

Theorem 6.4 ([42]). Let $C \subset\left(T^{*} X \backslash 0\right) \times\left(T^{*} Y \backslash \widetilde{0}\right)$ be a smooth complex positive homogeneous canonical relation, closed in $T^{*}(\widehat{X \times Y}) \backslash 0$. Assume that the complex smooth factorization condition (CSF) is satisfied. Let $\mu=-k|1 / p-1 / 2|$ and $1<p<$ $\infty$. Then Fourier integral operators of order $\mu$ with complex phases and canonical relation $C$ are continuous from $L_{\text {comp }}^{p}(Y)$ to $L_{\text {loc }}^{p}(X)$.

Theorem 6.4 deals with operators with symbols of type $(1,0)$. If the amplitude of a Fourier integral operator with the canonical relation as in Theorem 6.4 is in the class $S_{\rho, 1-\rho}^{\mu}, \rho \geq 1 / 2$, then it is continuous from $L_{\text {comp }}^{p}(Y)$ to $L_{l o c}^{p}(X)$ provided that $\mu=-(k-(n-k)(1-\rho))|1 / p-1 / 2|$.

There are close relations between the regularity properties of Fourier integral operators with complex phases to the symplectic and analytic geometries, in particular to singularities of the so-called affine fibrations. We refer to [41] for details.

\section{Global $L^{2}$ estimates for Fourier integral operators}

We now consider globally defined Fourier integral operators on $\mathbb{R}^{n}$ defined by

$$
T u(x)=\int_{\mathbb{R}^{n}} \int_{\mathbb{R}^{n}} e^{i(x \cdot \xi+\phi(y, \xi))} a(x, y, \xi) u(y) d \xi d y,
$$

where real-valued phase function $\phi \in C^{\infty}\left(\mathbb{R}^{n} \times \mathbb{R}^{n}\right)$ will satisfy conditions specified below. We note that in the case of pseudo-differential operators we have

$$
\phi(y, \xi)=-y \cdot \xi \text {. }
$$

Similar results will hold for the adjoint operator. In particular, this includes operators of the form

$$
S u(x)=\int_{\mathbb{R}^{n}} e^{i \phi(x, \xi)} a(x, \xi) \widehat{u}(\xi) d \xi,
$$

which appear as propagators for some classes of hyperbolic equations.

Global properties on $L^{2}\left(\mathbb{R}^{n}\right)$ of pseudo-differential operators are well-known (see e.g. Calderon-Vaillancourt [8], Cordes [12], Coifman-Meyer [10], Childs [9], Sugimoto [58], Boulkhemair [6], and many other contributions).

The case of pseudo-differential operators was also studied but much less is known (see Asada-Fujiwara [3, Asada [1, 2], Fujiwara [21, Kumano-go [27, Boulkhemair [7]). A certain disadvantage of these results is that in all these papers the authors made an assumption that $\partial_{\xi} \partial_{\xi} \phi$ is globally bounded on $\mathbb{R}^{n} \times \mathbb{R}^{n}$, which fails in many important situations.

For example, in a typical application to smoothing problems (as in [48]-[52]), the canonical transforms used for the changes of variables on the Fourier transform side 
have the phase of the form $x \cdot \xi-y \cdot \psi(\xi)$, in which case we have $\phi(y, \xi)=y \cdot \psi(\xi)$, where $\psi$ is positively homogeneous of order one. But then

$$
\partial_{\xi} \partial_{\xi} \phi(y, \xi)=y \cdot \partial_{\xi} \partial_{\xi} \psi(\xi)
$$

is unbounded on $\mathbb{R}^{n} \times \mathbb{R}^{n}$.

There are several questions that arise. For example, what are the minimal growth conditions on the phase and amplitude for operators (7.1) to be globally bounded on $L^{2}\left(\mathbb{R}^{n}\right)$. Moreover, if we want to have weighted estimates in $L^{2}\left(\mathbb{R}^{n}\right)$, or weighted estimates in Sobolev spaces over $L^{2}\left(\mathbb{R}^{n}\right)$, the question arises of what are the minimal requirements for the global calculus of such operators.

Let us now assume that the phase satisfies the following conditions on $\operatorname{supp} a$ :

$$
\begin{aligned}
& \left|\operatorname{det} \partial_{y} \partial_{\xi} \phi(y, \xi)\right| \geq C>0, \quad \forall(y, \xi) \in \mathbb{R}^{n} \times \mathbb{R}^{n} ; \\
& \left|\partial_{y}^{\alpha} \partial_{\xi} \phi(y, \xi)\right| \leq C_{\alpha},\left|\partial_{y} \partial_{\xi}^{\beta} \phi(y, \xi)\right| \leq C_{\beta} \\
& \forall(y, \xi) \in \mathbb{R}^{n} \times \mathbb{R}^{n}, \quad 1 \leq|\alpha|,|\beta| \leq 2 n+2 .
\end{aligned}
$$

Note that condition (C1) is a global version of the local graph condition, which is necessary even for local $L^{2}$-bounds for operators with amplitudes in $S_{1,0}^{0}$.

The importance of condition (C2) is that now we must take only mixed derivatives with respect to $y$ and $\xi$, so the phase functions for the canonical transforms in smoothing problems satisfy this condition. Indeed, if $\phi(y, \xi)=y \cdot \psi(\xi)$, where $\psi$ is homogeneous of order one for large $\xi$ and $|\operatorname{det} D \psi(\xi)| \geq C>0$, then condition $(\mathrm{C} 2)$ is satisfied for large frequencies. An additional argument is required for small frequencies and it can be found in 50.

It can be noted that conditions $(\mathrm{C} 1)$ and $(\mathrm{C} 2)$ are considerably weaker than those appearing in the analysis of SG-pseudo-differential operators (see Cordes [13]), SGFourier integral operators, (see Coriasco [14]), or for operator of Shubin type (see Boggiatto, Buzano and Rodino [5]).

We consider first operators of the form

$$
T u(x)=\int_{\mathbb{R}^{n}} \int_{\mathbb{R}^{n}} e^{i(x \cdot \xi+\phi(y, \xi))} a(x, \xi) u(y) d \xi d y .
$$

For such operators we have the following theorem

Theorem $7.1([50])$. Let $\phi(y, \xi)$ satisfy conditions (C1), (C2). Let a $(x, \xi)$ satisfy one of the following conditions:

(1)[Calderón-Vaillancourt] $\partial_{x}^{\alpha} \partial_{\xi}^{\beta} a(x, \xi) \in L^{\infty}\left(\mathbb{R}_{x}^{n} \times \mathbb{R}_{\xi}^{n}\right), \alpha, \beta \in\{0,1\}^{n}$.

(2) $\left[\right.$ Cordes] $\partial_{x}^{\alpha} \partial_{\xi}^{\beta} a(x, \xi) \in L^{\infty}\left(\mathbb{R}_{x}^{n} \times \mathbb{R}_{\xi}^{n}\right),|\alpha|,|\beta| \leq[n / 2]+1$.

(3) $\left[\right.$ Cordes] $\exists \lambda, \lambda^{\prime}>n / 2:\left(1-\Delta_{x}\right)^{\lambda / 2}\left(1-\Delta_{\xi}\right)^{\lambda^{\prime} / 2} a(x, \xi) \in L^{\infty}\left(\mathbb{R}_{x}^{n} \times \mathbb{R}_{\xi}^{n}\right)$.

(4) [Childs] difference conditions to be found in [50].

(5)[Coifman-Meyer] $\partial_{x}^{\alpha} \partial_{\xi}^{\beta} a(x, \xi) \in L^{\infty}\left(\mathbb{R}_{x}^{n} \times \mathbb{R}_{\xi}^{n}\right),|\alpha| \leq[n / 2]+1, \beta \in\{0,1\}^{n}$.

(6)[Coifman-Meyer] $\exists 2 \leq p<\infty$ : $\partial_{x}^{\alpha} \partial_{\xi}^{\beta} a(x, \xi) \in L^{p}\left(\mathbb{R}_{x}^{n} \times \mathbb{R}_{\xi}^{n}\right),|\alpha| \leq[n(1 / 2-$ $1 / p)]+1,|\beta| \leq 2 n$.

Then $T$ is $L^{2}\left(\mathbb{R}^{n}\right)$-bounded.

In brackets we list the names of the authors of the corresponding results for pseudodifferential operators. This theorem follows from a more general statement for the 
$L^{2}$-boundedness of Fourier integral operators with symbols in Besov spaces that appeared in [50].

There are also global $L^{2}$-boundedness theorems for adjoint operators, as well as for Fourier integral operators with general amplitudes. We refer to [50] for details of all such statements, as well as for results in weighted $L^{2}\left(\mathbb{R}^{n}\right)$ spaces.

The global calculus of operators (7.1) as well as global boundedness theorems in weighted Sobolev spaces can be found in [51].

\section{Fourier integral operators in Colombeau's spaces}

In this section we will discuss properties of Fourier integral operators in the Colombeau's spaces of new generalised functions. For details on these spaces we refer to e.g. [11] or [34.

The theory of pseudo-differential operators in Colombeau's spaces has been developed in [18] and [19]. Elements of the corresponding theory of Fourier integral operators has been laid down in [20].

At the same time, hyperbolic partial differential equations in Colombeau's spaces have been studied in [28], 25] by the energy methods, yielding relevant extensions of $L^{2}$ type results to the setting of new generalised functions.

In this section we will present corresponding $L^{p}$-results in the setting of Colombeau's generalised functions, for Fourier integral operators, with subsequent corresponding implications for solutions to hyperbolic equations.

Let $X$ be a bounded open set. Consider families $\left(u_{\epsilon}\right)_{\epsilon}$ of functions $u_{\epsilon} \in L_{\infty}^{p}(X)$, $0<\epsilon \leq 1$, where $L_{\infty}^{p}(X)$ stands for the space of function in $L^{p}(X)$ for which all derivatives also belong to $L^{p}(X)$. One can single out several important families of such functions dependent on their behaviour with respect to $\epsilon$. Thus, the class of moderate families $\mathcal{E}_{L^{p}}(X)$ is defined as the collection of families satisfying

$$
\forall \alpha \geq 0 \exists N \geq 0:\left\|\partial^{\alpha} u_{\epsilon}\right\|_{L^{p}}=O\left(\epsilon^{-N}\right) \text { as } \epsilon \rightarrow 0 .
$$

The class of null families $\mathcal{N}_{L^{p}}(X)$ is defined as a subclass of $\mathcal{E}_{L^{p}}(X)$ satisfying the condition that

$$
\forall N \geq 0:\left\|u_{\epsilon}\right\|_{L^{p}}=O\left(\epsilon^{N}\right) \text { as } \epsilon \rightarrow 0 .
$$

The Colombeau's algebra $\mathcal{G}_{L^{p}}(X)$ is then defined as

$$
\mathcal{G}_{L^{p}}(X):=\mathcal{E}_{L^{p}}(X) / \mathcal{N}_{L^{p}}(X) .
$$

Distributions $L_{-\infty}^{p}=\bigcup_{s \in \mathbb{R}} L_{s}^{p}$ are embedded in $\mathcal{G}_{L^{p}}(X)$ by the mapping $\iota(u)=[(u *$ $\left.\left(\rho_{\epsilon}\right)\right]_{\epsilon}$, where $\rho_{\epsilon}(x)=\epsilon^{-n} \rho(x / \epsilon)$ is the standard Friedrichs mollifier.

Subsequently, one can define the corresponding classes of generalized pseudo-differential operators. Thus, the generalized symbol is defined as a family $\left(a_{\epsilon}\right)_{\epsilon}$ of usual symbols $a_{\epsilon} \in S^{m}$ such that

$$
\forall k, l \exists N: \sup _{|\alpha| \leq k,|\beta| \leq l} \sup _{x, \xi \in \mathbb{R}^{n}}(1+|\xi|)^{-m+|\alpha|}\left|\partial_{\xi}^{\alpha} \partial_{x}^{\beta} a_{\epsilon}(x, \xi)\right|=O\left(\epsilon^{-N}\right) \text { as } \epsilon \rightarrow 0 .
$$

Then $A: \mathcal{G}_{L^{p}}(X) \rightarrow \mathcal{G}_{L^{p}}(X)$ is a generalised pseudo-differential operator with generalised symbol $\left(a_{\epsilon}\right)_{\epsilon}$ if, on the representative level, it acts as

$$
\left(u_{\epsilon}\right)_{\epsilon} \mapsto\left(a_{\epsilon}\left(t, x, D_{x}\right) u_{\epsilon}\right)_{\epsilon}
$$


To formulate the results, also the notion of the slow scale is required. A generalised symbol $\left(a_{\epsilon}\right)_{\epsilon}$ is said to be of log-type up to order $(k, l)$ (or to be slow scale) if

$$
\sup _{|\alpha| \leq k,|\beta| \leq l} \sup _{x, \xi \in \mathbb{R}^{n}}(1+|\xi|)^{-m+|\alpha|}\left|\partial_{\xi}^{\alpha} \partial_{x}^{\beta} a_{\epsilon}(x, \xi)\right|=O(\log (1 / \epsilon)) \text { as } \epsilon \rightarrow 0 .
$$

In [28], Lafon and Oberguggenberger considered partial differential operators of the form

$$
A=\sum_{j=1}^{n} a_{j}(t, x) \partial_{x_{j}}+b(t, x) .
$$

They investigated the Cauchy problem for operator $D_{t}+A$ and showed the existence of solutions in $\mathcal{G}_{L^{\infty}}(X)$ provided that $b$ and $\partial_{x_{k}} a_{j}$ are of $\log$-type. Moreover, if $a_{j}$ and $b$ are constant for large $x$, then the solution is also unique, and in [35] an example of the non-uniqueness was given if this condition breaks. In [25], Hörmann considered the Cauchy problem for more general pseudo-differential operators $A$ of log-type, for which he showed existence and uniqueness in $\mathcal{G}_{L^{2}}(X)$ (also giving some estimates on $k$ and $l$ in the log-type assumption). In particular, the non-uniqueness effect disappears in $\mathcal{G}_{L^{2}}(X)$ compared to $\mathcal{G}_{L^{\infty}}(X)$.

These results may provide some hints on the behaviour of relevant Fourier integral operators in Colombeau' spaces. Let us consider generalised Fourier integral operators of the form

$$
T u(x)=\int_{X} \int_{\mathbb{R}^{n}} e^{i \phi(x, y, \xi)} a(x, y, \xi) u(y) d y d \xi .
$$

Let us define the regular Colombeau's algebra. The class of regular families $\mathcal{R}_{L^{p}}(X)$ is defined as a class of functions satisfying the condition

$$
\exists N \geq 0 \forall \alpha \geq 0:\left\|\partial^{\alpha} u_{\epsilon}\right\|_{L^{p}}=O\left(\epsilon^{-N}\right) \text { as } \epsilon \rightarrow 0 .
$$

Then the regular Colombeau algebra $\mathcal{G}_{L^{p}}^{\infty}(X)$ is defined by

$$
\mathcal{G}_{L^{p}}^{\infty}(X):=\mathcal{R}_{L^{p}}(X) / \mathcal{N}_{L^{p}}(X) .
$$

In [20], Garetto, Hörmann and Oberguggenberger showed that if $\phi$ is a non-degenerate phase function then $T$ maps $\mathcal{G}_{L^{\infty}}(X)$ to itself continuously. If the phase function $\phi$ is a generalised family satisfying the slow scale assumption (e.g. log-type), and $a=\left(a_{\epsilon}\right)_{\epsilon}$ is a regular family of amplitudes, then $T$ maps locally continuously the space of regular Colombeau's functions $\mathcal{G}_{L^{\infty}}^{\infty}(X)$ to itself. The following theorem extends this to Colombeau's algebras over any $L^{p}(X), 1 \leq p \leq \infty$.

Theorem 8.1. Let $1 \leq p \leq \infty$. If $\phi$ is a non-degenerate (generalised) phase function then $T$ maps $\mathcal{G}_{L^{p}}(X)$ to itself continuously. If the phase function $\phi$ is a generalised family satisfying the slow scale assumption (e.g. log-type), and $a=\left(a_{\epsilon}\right)_{\epsilon}$ is a regular family of amplitudes, then $A$ maps locally continuously the space of regular Colombeau's generalised functions $\mathcal{G}_{L^{p}}^{\infty}(X)$ to itself.

Consequently, propagators for strictly hyperbolic Cauchy problems are continuous in $\mathcal{G}_{L^{p}}(X)$, and in $\mathcal{G}_{L^{p}}^{\infty}(X)$ under the log-type assumption on the phase, for all $1 \leq$ $p \leq \infty$.

The proof is based on the extension to the Colombeau's setting of the eikonal and transport equations, modulo controllable errors with respect to $\epsilon$, and on estimates 
for generalised Fourier integral operators in $\mathcal{G}_{L^{p}}(X)$. Details of proofs and exact losses of regularity in Colombeau's spaces will appear in [46].

\section{REFERENCES}

[1] K. Asada, On the $L^{2}$ boundedness of Fourier integral operators in $\mathbb{R}^{n}$. Proc. Japan Acad. Ser. A Math. Sci. 57 (1981), 249-253.

[2] K. Asada, On the $L^{2}$ boundedness theorem of nonhomogeneous Fourier integral operators in $\mathbb{R}^{n}$. Kodai Math. J. 7 (1984), 248-272.

[3] K. Asada and D. Fujiwara, On some oscillatory integral transformations in $L^{2}\left(\mathbb{R}^{n}\right)$. Japan. J. Math. (N.S.) 4 (1978), 299-361.

[4] M. Beals, $L^{p}$ boundedness of Fourier integrals. Mem. Amer. Math. Soc. 264 (1982).

[5] P. Boggiato, E. Buzano and L. Rodino. Global hypoellipticity and spectral theorey. Akademie Verlag, Berlin, 1996.

[6] A. Boulkhemair, $L^{2}$ estimates for pseudodifferential operators. Ann. Scuola Norm. Sup. Pisa Cl. Sci. 22 (1995), 155-183.

[7] A. Boulkhemair, Estimations $L^{2}$ precisees pour des intégrales oscillantes. Comm. Partial Differential Equations 22 (1997), 165-184.

[8] A. P. Calderón and R. Vaillancourt, On the boundedness of pseudo-differential operators, J. Math. Soc. Japan 23 (1971), 374-378.

[9] A. G. Childs, On the $L^{2}$-boundedness of pseudo-differential operators, Proc. Amer. Math. Soc. 61 (1976), 252-254.

[10] R. R. Coifman, Y. Meyer, Au-delà des opérateurs pseudo-différentiels, Astérisque 57 (1978).

[11] J.-F. Colombeau, New generalized functions and multiplication of distributions. North-Holland Publishing Co., 1984.

[12] H. O. Cordes, On compactness of commutators of multiplications and convolutions, and boundedness of pseudodifferential operators, J. Funct. Anal. 18 (1975), 115-131.

[13] H. O. Cordes, The technique of pseudodifferential operators, Cambridge Univ. Press, 1995.

[14] S. Coriasco, Fourier integral operators in SG classes I: composition theorems and action on SG Sobolev spaces, Rend. Sem. Mat. Univ. Pol. Torino 57 (1999), 249-302.

[15] J.J. Duistermaat, Fourier integral operators. Birkhäuser, Boston, 1996.

[16] Yu. V. Egorov, Microlocal analysis. Encyclopedia Math. Sci., Partial Differential Equations, IV, 33, Springer, 1993, 1-147.

[17] G. I. Eskin, Degenerate elliptic pseudo-differential operators of principal type, Math. USSR Sbornik, 11 (1970), 539-585.

[18] C. Garetto, T. Gramchev and M. Oberguggenberger, Pseudodifferential operators with generalized symbols and regularity theory. Electron. J. Differential Equations 116 (2005).

[19] C. Garetto, G. Hörmann, Microlocal analysis of generalized functions: pseudodifferential techniques and propagation of singularities. Proc. Edinb. Math. Soc. 48 (2005), 603-629.

[20] C. Garetto, G. Hörmann and M. Oberguggenberger, Generalized oscillatory integrals and Fourier integral operators, arXiv:math/0607706.

[21] D. Fujiwara, On the boundedness of integral transformations with highly oscillatory kernels, Proc. Japan Acad. 51 (1975), 96-99.

[22] L. Hörmander, Fourier integral operators. I, Acta Math. 127 (1971), 79-183.

[23] L. Hörmander, $L^{2}$ estimates for Fourier integral operators with complex phase. Arkiv för Matematik 21 (1983), 283-307.

[24] L. Hörmander, The analysis of linear partial differential operators. Vols. III-IV, Springer-Verlag, New York, Berlin, 1985.

[25] G. Hörmann, First-order hyperbolic pseudodifferential equations with generalized symbols. J. Math. Anal. Appl. 293 (2004), 40-56.

[26] I. Kamotski, M. Ruzhansky, Regularity properties, representation of solutions and spectral asymptotics of systems with multiplicities. Comm. Partial Differential Equations 32 (2007), 1-35.

[27] H. Kumano-go, A calculus of Fourier integral operators on $\mathbb{R}^{n}$ and the fundamental solution for an operator of hyperbolic type, Comm. Partial Differential Equations 1 (1976), 1-44. 
[28] F. Lafon, M. Oberguggenberger, Generalized solutions to symmetric hyperbolic systems with discontinuous coefficients: the multidimensional case. J. Math. Anal. Appl. 160 (1991), 93-106.

[29] A. Laptev, Yu. Safarov and D. Vassiliev, On global representation of Lagrangian distributions and solutions of hyperbolic equations. Comm. Pure Appl. Math. 47 (1994), 1411-1456.

[30] P. Lax, Asymptotic solutions of oscillatory initial value problems. Duke Math. J. 24 (1957), 627-646.

[31] A. Melin, J. Sjöstrand, Fourier integral operators with complex-valued phase functions. Springer Lecture Notes 459 (1975), 120-223.

[32] A. Melin, J. Sjöstrand, Fourier integral operators with complex phase functions and parametrix for an interior boundary problem. Comm. Partial Differential Equations 1 (1976), 313-400.

[33] A. Miyachi, On some estimates for the wave operator in $L^{p}$ and $H^{p}$. J. Fac. Sci. Univ. Tokyo Sect. IA Math. 27 (1980), 331-354.

[34] M. Nedeljkov, S. Pilipović and D. Scarpalézos, The linear theory of Colombeau generalized functions. Pitman Research Notes in Mathematics Series, 1998.

[35] M. Oberguggenberger, Hyperbolic systems with discontinuous coefficients: generalized solutions and a transmission problem in acoustics. J. Math. Anal. Appl. 142 (1989), 452-467.

[36] J. Peral, $L^{p}$ estimates for the wave equation. J. Funct. Anal. 36 (1980), 114-145.

[37] D.H. Phong, Regularity of Fourier integral operators. Proc. Int. Congress Math., Zürich, Switzerland, 1994, 862-874.

[38] G. Rozenblum, Spectral asymptotic behavior of elliptic systems. (Russian) Zap. LOMI, 96 (1980), 255-271.

[39] M. Ruzhansky, On the sharpness of Seeger-Sogge-Stein orders. Hokkaido Math. J. 28 (1999), $357-362$.

[40] M. Ruzhansky, Analytic Fourier integral operators, Monge-Ampere equation and holomorphic factorization. Arch. Math. 72 (1999), 68-76.

[41] M. V. Ruzhansky, Singularities of affine fibrations in the regularity theory of Fourier integral operators. Russian Math. Surveys 55 (2000), 99-170.

[42] M. Ruzhansky, Regularity theory of Fourier integral operators with complex phases and singularities of affine fibrations. CWI Tract, volume 131, 2001.

[43] M. V. Ruzhansky, Regularity of the parametrix of a problem with oblique derivative. Russian Math. Surveys 56 (2001), 1179-1180.

[44] M. Ruzhansky, On the failure of the factorization condition for non-degenerate Fourier integral operators. Proc. of Amer. Math. Soc. 130 (2002), 1371-1376.

[45] M. Ruzhansky, Recent progress in the regularity theory of Fourier integrals with real and complex phases and solutions to partial differential equations. Banach Center Publications, 60 (2003), $151-160$.

[46] M. Ruzhansky, On the properties of Fourier integral operators in spaces of new generalised functions, in preparation.

[47] M. Ruzhansky, J. Smith, Global time estimates for higher order hyperbolic equations. Journees "Equations aux Derivees Partielles", Exp. No. XII, 29 pp., Ecole Polytech., Palaiseau, 2005.

[48] M. Ruzhansky, M. Sugimoto, New proof of global smoothing estimates for dispersive equations. Operator Theory: Advances and Applications 155 (2004), 65-75.

[49] M. Ruzhansky, M. Sugimoto, A smoothing property of Schrödinger equations in the critical case. Math. Ann. 335 (2006), 645-673.

[50] M. Ruzhansky, M. Sugimoto, Global $L^{2}$ boundedness theorems for a class of Fourier integral operators. Comm. Partial Differential Equations 31 (2006), 547-569.

[51] M. Ruzhansky, M. Sugimoto, Global calculus of Fourier integral operators, weighted estimates, and applications to global analysis of hyperbolic equations. Operator Theory: Advances and Applications 164 (2006), 65-78.

[52] M. Ruzhansky, M. Sugimoto, Global boundedness theorems for Fourier integral operators associated with canonical transformations. Harmonic analysis and its applications, 65-75, Yokohama Publ., Yokohama, 2006. 
[53] Yu. Safarov, D. Vassiliev, The asymptotic distribution of eigenvalues of partial differential operators. American Mathematics Society, 1996.

[54] A. Seeger, C.D. Sogge and E.M. Stein, Regularity properties of Fourier integral operators. Ann. of Math. 134 (1991), 231-251.

[55] C.D. Sogge, Fourier integrals in classical analysis. Cambridge University Press, 1993.

[56] E. M. Stein, $L^{p}$ boundedness of certain convolution operators. Bull. Amer. Math. Soc. 77 (1971), 404-405.

[57] E.M. Stein, Harmonic analysis. Princeton University Press, Princeton, 1993.

[58] M. Sugimoto, $L^{2}$-boundedness of pseudo-differential operators satisfying Besov estimates I, J. Math. Soc. Japan 40 (1988), 105-122.

[59] M. Sugimoto, On some $L^{p}$-estimates for hyperbolic equations. Arkiv för Matematik 30 (1992), 149-162.

[60] T. Tao, The weak-type $(1,1)$ of Fourier integral operators of order $-(n-1) / 2$. J. Aust. Math. Soc. 76 (2004), 1-21.

[61] F. Treves, Introduction to pseudodifferential and Fourier integral operators. Vol. 2, Plenum Press, 1982.

Department of Mathematics, Imperial College London, United Kingdom

E-mail address: m.ruzhansky@imperial.ac.uk 